\title{
Une formation continue taillée sur mesure
}

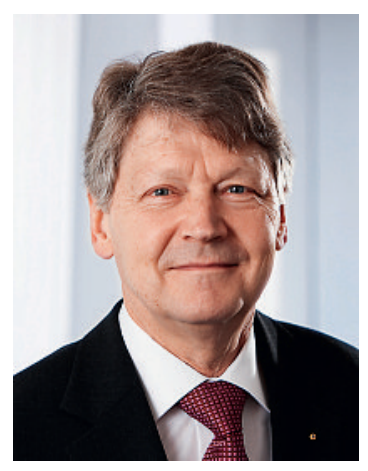

Le corps médical suisse continue de se former régulièrement. Depuis le $1^{\mathrm{er}}$ septembre 2007, date d'entrée en vigueur de la loi sur les professions médicales (LPMéd), la formation continue fait partie des devoirs professionnels de tous les médecins exerçant en Suisse. Lorsqu'ils ont accompli le programme de formation continue d'une société de discipline, ils obtiennent un diplôme leur servant d'attestation, notamment face aux patients, aux autorités et aux assureurs. Dans la présente édition du Bulletin des médecins suisses, Christoph Hänggeli, qui est fixée dans chaque programme de formation continue. Par ailleurs, un catalogue détaillé d'objectifs contraignants pour la formation continue conduirait à une sur-réglementation massive et incontrôlable.

Le diplôme de formation continue mis au point conjointement par les sociétés de discipline médicale et l'ISFM est réservé aux membres de la FMH. Les non-membres se voient remettre une attestation équivalente.

Le contrôle des protocoles de formation continue incombe aux sociétés de discipline médicale; selon leurs estimations, près de $97 \%$ des médecins satisfont aux prescriptions du programme de formation continue concerné et disposent des 150 crédits qui doivent être acquis en trois ans. A l'instar des pays qui nous entourent, nous refusons d'élargir ce contrôle et d'instaurer une évaluation sommative des connaissances ou capacités de chaque médecin, comme c'est

\section{Afin de simplifier les tâches administratives nécessaires à l'obtention des diplômes de formation continue, I'ISFM a initié la création d'un portail de la formation continue basé sur l'internet}

administrateur responsable de l'ISFM, expose la valeur et l'utilité du diplôme de formation continue pour les médecins [1]. Ce diplôme certifie d'une part qu'ils ont accompli la formation continue exigée par la loi et les habilite, d'autre part, à facturer leurs prestations selon le TARMED.

\section{Ce diplôme certifie d'une part qu'ils ont accompli la formation continue exigée par la loi et les habilite, d'autre part, à facturer leurs prestations selon le TARMED}

Les médecins soumis à cette obligation devront déterminer eux-mêmes la formation continue dont ils ont besoin dans le cadre de leur activité actuelle. Les moyens, les contenus et la méthodologie découlent ensuite de ce choix. Le listage des différentes capacités individuelles en matière d'examen médical ou d'intervention médicale n'est pas possible dans ce contexte; c'est donc la seule durée de la formation le cas en Grande-Bretagne et dans les Etats nord-américains [2] à plus ou moins grande échelle. En revanche, le groupe de travail de l’ISFM «Soutien des sociétés de discipline médicale dans la formation continue» envisage une évaluation formative dans le cadre d'un portfolio de formation continue individuel. Afin de simplifier les tâches administratives nécessaires à l'obtention des diplômes de formation continue, l'ISFM a initié la création d'un portail de la formation continue basé sur l'internet [3]. A l'avenir, les médecins pourront attester leur formation continue au moyen d'un diplôme sans devoir effectuer des démarches administratives fastidieuses.

Dr Max Giger, président de l'ISFM

\section{Références:}

1 Hänggeli C. Possédez-vous un diplôme de formation continue? Bull Méd Suisses. 2009;90(49):1906-8.

2 Shaw K, Cassel CK et al. Shared Medical Regulation in a Time of Increasing Calls for Accountability and Transparency. JAMA. 2009;302:2008-14.

3 Giger M, Hänggeli C. ISFM: principaux projets. Bull Méd Suisses. 2009;90(26/27):1034-36. 